\title{
Estimation of Arm Joint Angles from Surface Electromyography signals using Artificial Neural Networks
}

\author{
Sauvik Das Gupta ${ }^{1}$, Sourav Das ${ }^{2}$, Partha Das ${ }^{3}$, SK Masud Hossain ${ }^{4}$, \\ Subhajit Ghosh ${ }^{5}$, Shanta Mazumder ${ }^{6}$ \\ ${ }^{1}$ School of Electrical and Computer Engineering, Oklahoma State University, Stillwater, OK, USA \\ ${ }^{23456}$ West Bengal University of Technology, Kolkata, West Bengal, India
}

\begin{abstract}
Vicon system is implemented in almost every motion analysis systems. It has many applications like robotics, gaming, virtual reality and animated movies. The motion and orientation plays an important role in the above mentioned applications. In this paper we propose a method to estimate arm joint angles from surface Electromyography (s-EMG) signals using Artificial Neural Network (ANN). The neural network is trained with $E M G$ data from wrist flexion and extension action as input and joint angle values from the vicon system as target. The results shown in this paper illustrate the neural network performance in estimating the joint angle values during offline testing.
\end{abstract}

Index Terms: Vicon system, Joint angle, Surface EMG, Artificial Neural Network, Virtual reality, Robotics.

\section{Introduction}

The Vicon motion capture system is a state-of-the-art infrared marker-tracking system that offers millimeter resolution of 3D spatial displacements. The system we have implemented consists of twelve cameras outfitted with IR optical filters and an array of IR LEDs, and a set of reflective dots. The dots, arranged on the hand of the subject (human or non-human primate), reflect the IR radiation emitted by the LEDs. All other light is filtered so that the system only recognizes the dots. In software, the images taken from the twelve cameras are used to construct a three-dimensional representation of the markers.

Surface Electromyography (s-EMG) is a non-invasive technique for measuring muscle electrical activity that occurs during muscle contraction and relaxation cycles. EMG signals contain the information about the muscle force which can be used in human-machine interaction.

Much research has been done using electromyogram (EMG) signals and motion analysis for prosthetic arm. Ajiboyeet al. proposed a heuristic fuzzy logic approach to multiple electromyogram (EMG) pattern recognition for multifunctional prosthesis control [1]. Momenet al. proposed a real-time electromyography (EMG) classifier of user-selected intentional movements rather than an imposed subset of standard movements using Fuzzy C-means clustering [2]. Kuikenet al. assessed the performance of patients with upper-limb amputation who had undergone Targeted Muscle Re-innervation (TMR) surgery, using a pattern-recognition algorithm to decode EMG signals and control prosthetic-arm motions [3].Much research has been done in the field of predicting values from s-EMG signals [4]. Our objective is to estimate the joint angles from the EMG signals using regression techniques.

The next section describes the hardware setup of our system. Section III presents the methodology we proposed for joint angle estimation. Section IV discusses the experimental evaluation and results. Section V presents the conclusion of the paper.

\section{Hardware Platform}

The hardware platform of our research consists of aninstrumentation amplifier board, a data acquisition board, the vicon motion capture system, an EMG sensor anda sensor isolator. Below we will discuss in more details about the individual components.

\section{A. INA 326 Instrumentation Amplifier Board}

The bio-medical amplifier simulation is done in NI Multisim. The simulated circuit result is shown in the following figure 1. The EMG signals are basically in the range of microvolts. Hence we ensure that sufficientamplification is provided and the gain is quite high from this circuit so as to get the output voltage range in the millivolts. A gain of 1000 is thus selected. The Gain calculation is as follows:-

$\operatorname{Gain}(\mathrm{G})=2 *(\mathrm{R} 2 / \mathrm{R} 1)$ in the circuit $\longrightarrow(1)$

We choose $\mathrm{R} 2=1 \mathrm{MOhms}$ and $\mathrm{R}=2 \mathrm{kOhms}$ to get $\mathrm{G}=1000$

$\mathrm{G}=2 *(1,000,000 \mathrm{Ohms} / 2000 \mathrm{Ohms})=1000$ 


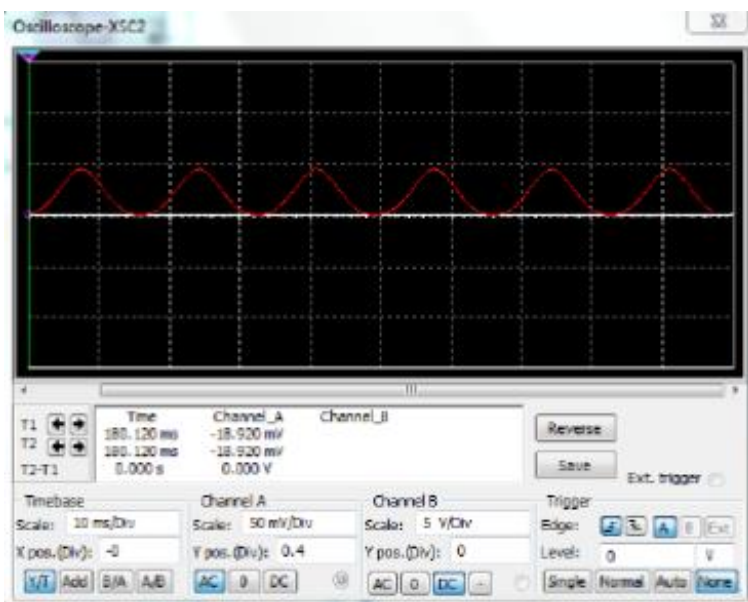

Figure 1: The simulated result in NI Multisim

We selected the INA 326 Instrumentation Amplifier because of the following properties:-

1. Precision

2. Low offset: $100 \mu \mathrm{V}$ (max)

3. Low offset drift: $0.4 \mu \mathrm{V} /{ }^{\circ} \mathrm{C}(\max )$

4. Excellent long-term stability

5. Very-low $1 / \mathrm{f}$ noise

6. True rail-to-rail I/O

7. Input common-mode range: $20 \mathrm{mV}$ below negative rail to $100 \mathrm{mV}$ above positive rail

8. Wide output swing: Within $10 \mathrm{mV}$ of rails

9. Supply range: single $+2.7 \mathrm{~V}$ to $+5.5 \mathrm{~V}$

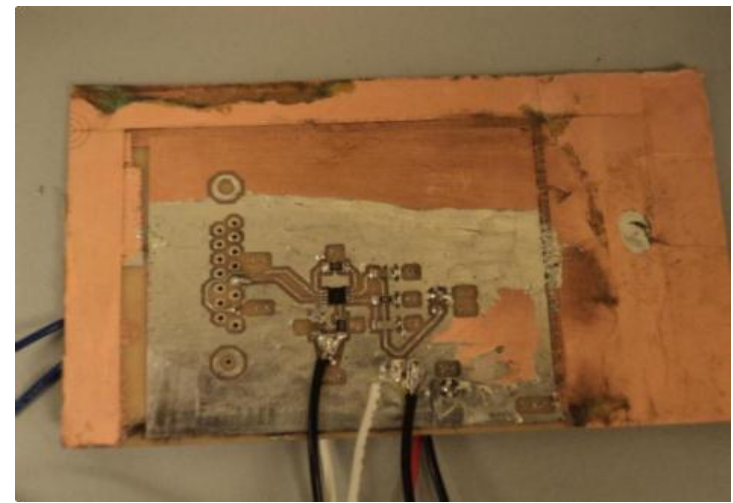

Figure 2: The INA 326 Amplifier Board

\section{B. Data Acquisition Board (NI-9234)}

The amplified s-EMG data is acquired by an NI-9234 Data Acquisition (USB-DAQ) device [5]. It is a C-series 4-channel Analog to Digital Convertor (ADC) DAQ manufactured by National Instruments, which provides BNC connectivity. This device is connected to a PC by a USB cable. It supports a sampling rate of $51200 \mathrm{samples} / \mathrm{sec}$ for each of the four channels. The input circuitry of the device consists of current limiting diodes, amplifier and pre-filter and an analog to digital converter (ADC). Some additional features supported by the device are AC/DC Coupling, Common Mode Bias Current and Integrated Electronics Piezoelectric (IEPE) sensors. Figure 3 shows the DAQ board. The EMG is sampled at 1700 samples/sec for this research through this device using theDAQ toolbox of MATLAB.

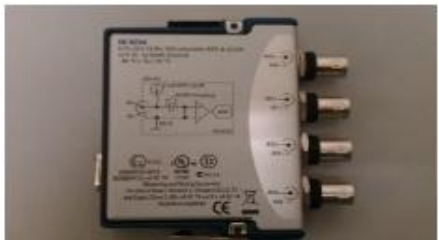

Figure 3: NI-9234 DAQ Board 


\section{Vicon Motion Capture System}

We use the Vicon Motion Capture System [6] in our lab to monitor the Joint Angles from the Human Arm. Infra-red Markers are placed on the arms and a set of 12 cameras monitor the position ofthe arm and give the Angle Feedback information. The Tracker software is used at first to define the rigid bodies made by the markers on the Arm and then MATLAB is used to stream the JointAngles directly to a PC through the LAN \& Internet.

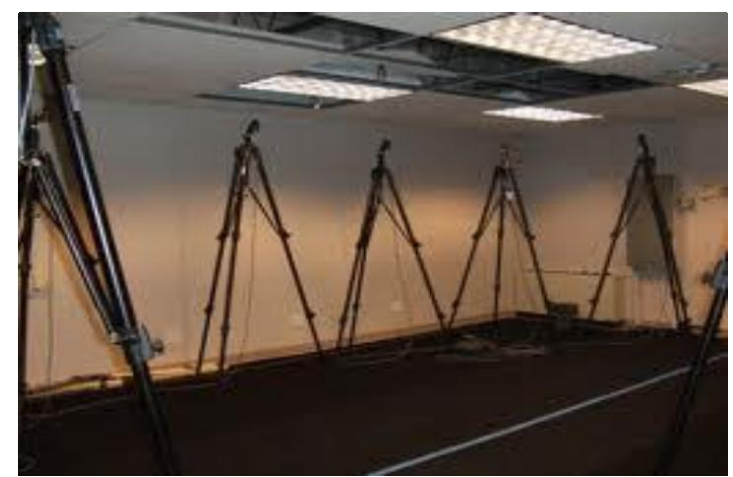

Figure 4: The Vicon Motion Capture System

\section{MyoScan-Z EMG Sensor (SE9503Z)}

We have used the INA 326 amplifier board during the first phase of the experiment then we switch to the MyoScan-Z EMG sensor for better results during the second phase of the experiment.

As shown in Figure 5(a), the MyoScan-Z EMG sensor [7] from Thought Technology Ltd., is used in our experiment. In order to measure, process and store EMG data, amplification is necessary. In the MyoScan-Z EMG sensor, adifferential amplifier schematic shown in Figure 5(b) is usedto amplify the EMG signals, where EMG signal is denoted by " $m$ " and the noise signal by " $n$ ". This amplifier has the abilityto reduce the noise and thus increase the resolution by increasing the Signal-to-Noise Ratio (SNR).

Some of the important specifications of the MyoScan-Z EMG sensor are as follows:

1. Designed for sampling rate of 2048 samples per second

2. Input impedance of $10^{12} \mathrm{ohms}$ in parallel with $10 \mathrm{pF}$

3. Measures raw EMG signals with a range from 0 up to $2000 \mathrm{mv}$.

4. Strong DC signal suppression

5. CMRR >130dB and input /output gain of 500 .

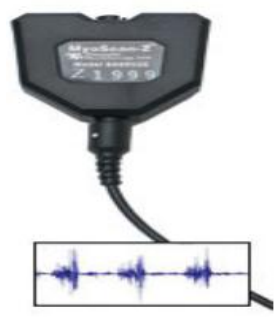

(a)

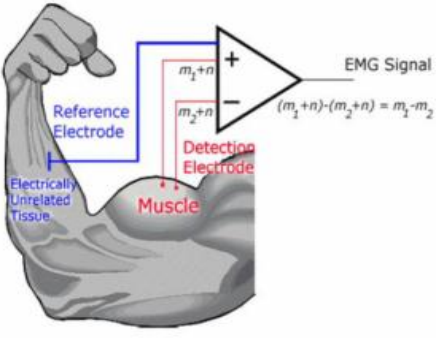

(b)

Figure 5: (a) MyoScan EMG Sensor, (b) Schematic of the differential amplifier configuration

\section{E. Sensor Isolator (T9405AM)}

In this project we also use a sensor isolator along with the MyoScan EMG Sensor. This is a 4-channel optical isolatorand the main motivation behind using this isolator is that itserves as an interface design, which provides electricalisolation of $4.5 \mathrm{kV}$. It also allows the sensor to be safelyinterfaced with the analog inputs of the line-powered systems,such as the DAQ card and the computer. Figure 6 shows thesensor isolator.

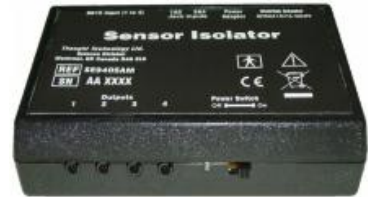

Figure 6: Sensor Isolator 


\section{III.Methodology}

The whole methodology can be divided into three distinct parts - first pre-processing of the EMG data, secondly training the ANN with EMG data as input and joint angles as the target and lastly offline testing of the ANN with anotherset of the EMG data. The pre-processing step includes feature extraction using root-meansquare (RMS) method. Then the data is smoothed using pseudo-Gaussian function and lastly normalization is applied onboth the EMG and joint angle data. Then the neural network is trained using the features of EMG and joint angle data as input and target respectively. Figure 7 shows a typical raw EMG signal.

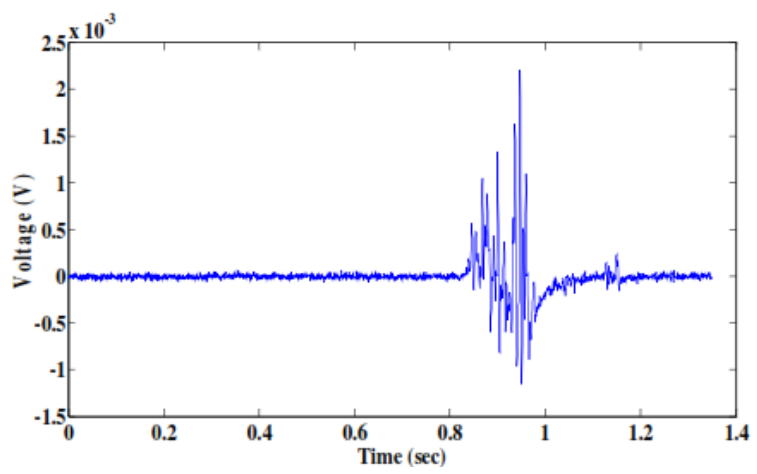

Figure 7: A typical raw EMG signal

\section{A.Pre-processing}

\section{i. Feature Extraction}

The Root-Mean-Square (RMS) is a popular method for feature extraction used in EMG pattern recognition. It removes the dual-axis nature of the EMG data. The mathematical equation for RMS is given as follows:-

$$
R M S=\sqrt{\left(\frac{1}{N}\right) \sum_{i=1}^{N} x_{i}^{2}}
$$

Where, $\mathrm{N}=$ Total number of values

$\mathrm{x}=$ Individual data points

A MATLAB script is coded for getting the dynamic RMS values of the EMG data using a continuous window update method throughout the length of the data. The value of the window $(\mathrm{N})$ is fixed to 30 samples in this case. Figure 8 shows the RMS plot of EMG data.

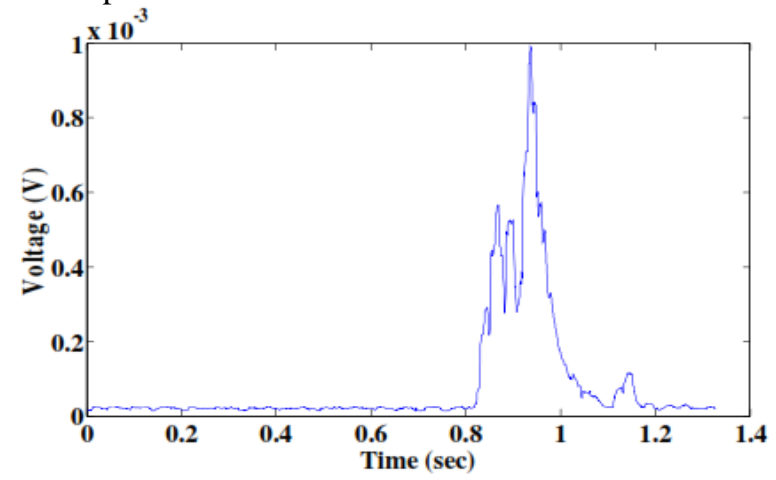

Figure 8: RMS plot of raw EMG signal

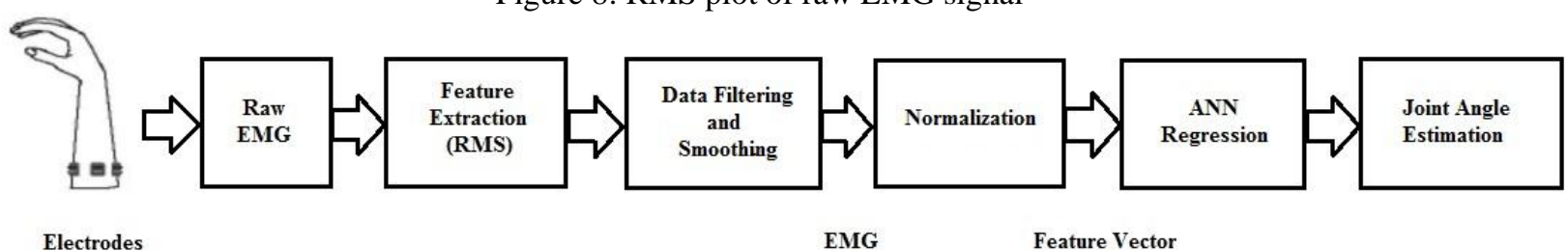

Figure 9: Proposed overall method for joint angle estimation 


\section{ii. Data Filtering and Smoothing}

The main objective in this part is to reduce noise thus increasing Signal-to-Noise (SNR) value. Raw EMG data needs to be filtered in order to improve the SNR. This is done by NI-9234 DAQ, since the device has hardware pre-filter which passes only in-band signals and attenuates any signal above stop-band frequency. Smoothing is applied on the signal to remove the high frequency fluctuations. Here we also implement a pseudo-Gaussian smoothing filter in MATLAB to achieve the above purpose additionally. It is observed that the noise is considerably reduced and the SNR is improved.

\section{iii. Normalization}

The s-EMG data is in millivolts $(\mathrm{mV})$ while the joint angle data is in steradian (sr). To achieve regression using neural network, both input and target is to be compared from a common frame of reference. This is done using a MATLAB function 'mapminmax' which scales inputs and targets so that they fall in range $[-1,1]$.

\section{B. Training the ANN}

The architecture of the ANN is a two layer feed-forward multi-layer perceptron network, with sigmoid activation function in the hidden layer and linear function in the output layer. The algorithm used is LevenbergMarquardt backpropagation algorithm [8] and Scaled Conjugate Gradient backpropagation algorithm [9]. One of the algorithms is selected by MATLAB depending upon optimum memory usage. The input to the network is the EMG feature vector and the joint angle data from the vicon system is the target vector.

Bias [10] is the measure of how good the model fits to the data. Variance is the measure of how much the prediction varies around its average. In order to improve the performance of the network and to prevent high bias and variance, the data is separated into training set, cross-validation set and testing set. The network is trained with two different sets of inputs (different sets of data obtained from a single channel of EMG) and targets (corresponding joint angle values).

The Mean Squared Error (MSE) is used for measuring the performance. Fifty hidden neurons yield good results. The average overall regression value from training, validation and test set is found to be 0.81555 (maximum value of $\mathrm{R}$ is 1 ).

\section{Offline testing}

The offline joint angle prediction method is introduced in the testing phase of the network. After the neural network is properly trained we acquire new sets of data for both EMG and joint angles. We process the EMG signal data using the above mentioned methods - RMS feature extraction, data filtering and smoothing as well as normalization. The normalized EMG data is then provided to the network as input and joint angles' data as target. Regression and performance measure are thereby noted and compared with their previous values.

\section{Experimental Evaluation}

We use the Kendall Arbo ECG/EEG/EMG electrodes [11] in this project as the sensing electrodes. First of all, the muscle sites are found out for the wrist flexion and extension actions. The flexor Carpi Ulnaris and the extensor Carpi Ulnaris muscles areidentified to be involved in these actions respectively. The selected hand area is cleaned with the NuPrep abrasive skin cream to remove the dead and dry cells in order to increase signal conduction and to minimize skin-electrode impedance. Ten20 conductive enhancement paste is applied on the grey portion of the EMG electrodes to enhance the conductivity of the electrodes. The MyoScan-Z EMG sensor is connected to the electrodes in order to acquire and amplify the EMG signals. The sensor isolator is used in conjunction with the EMG sensor as an isolation interface between the human subject and the line-powered DAQ device. Finally, the amplified EMG signals are acquired through the NI-9234 DAQ device into the computer through a MATLAB program. The acquired data is processed, trained and tested offline. Figures 10(a), 10(b), 10(c) and 10(d) show the predicted joint anglevalues from EMG signal against the measured joint angle values from the vicon system. The predicted as well as measured angles are plotted in figures 10(a), 10(b) and figures $10(\mathrm{c}), 10(\mathrm{~d})$ for flexion and extension respectively. This is done by acquiring the new data and predicting the joint angles from the EMG signal.Figures 11(a) and 11(b) represent regression plots for flexionand extension respectively. 


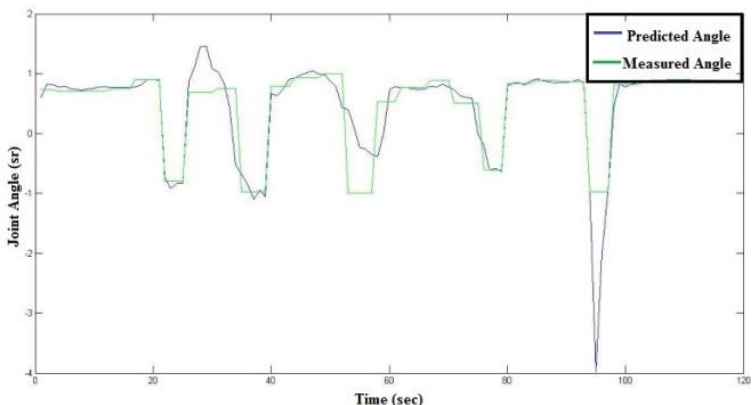

Figure 10(a): Estimated flexion angle value from EMG

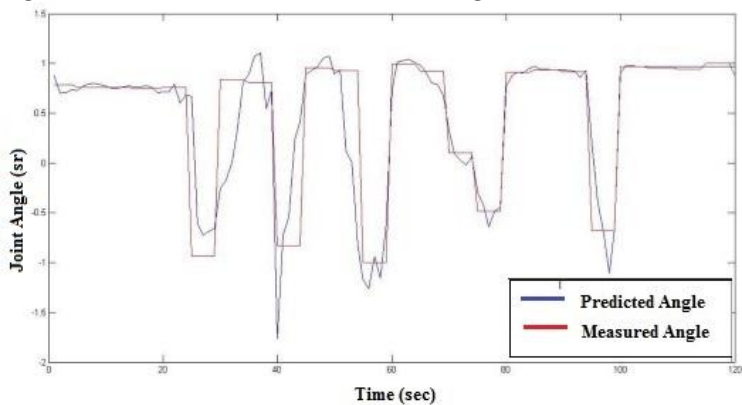

Figure 10(b): Estimated flexion angle value from EMG

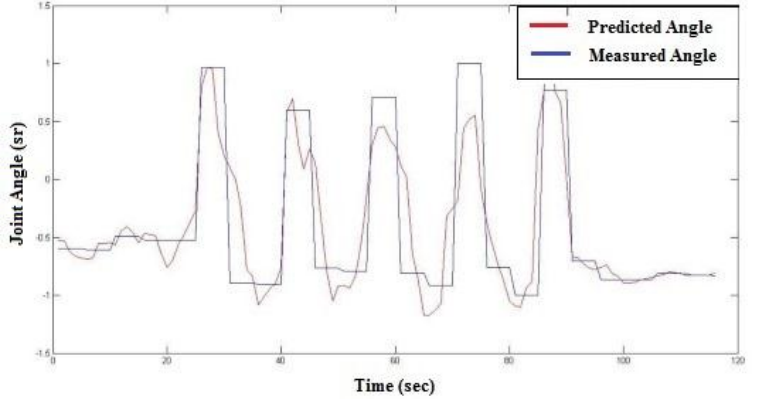

Figure 10(c): Estimated extension angle value from EMG

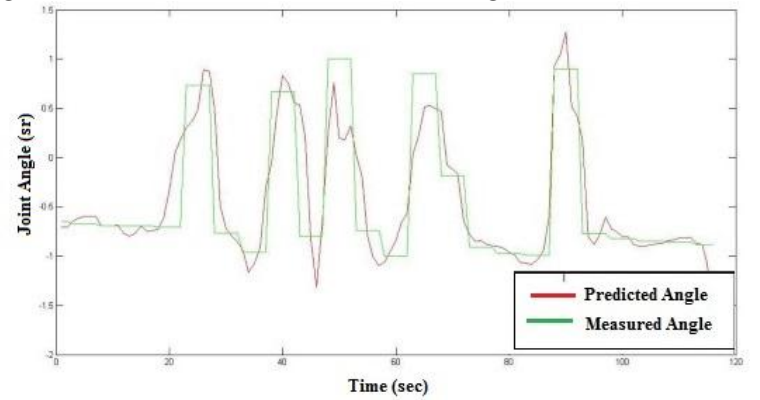

Figure 10(d): Estimated extension angle value from EMG

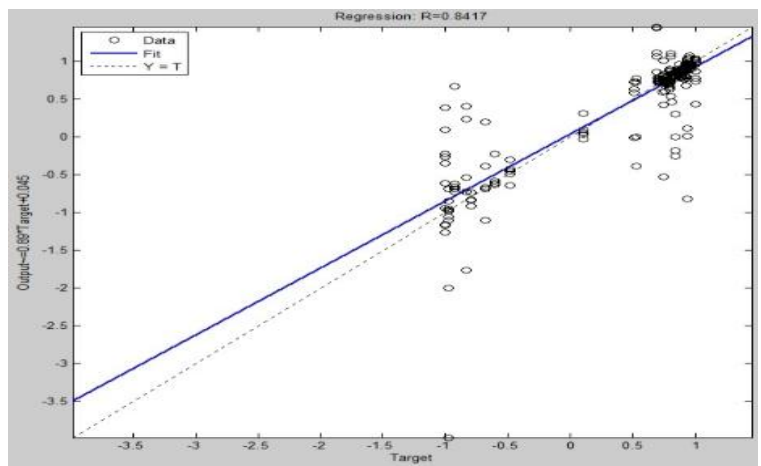

Figure 11(a): Regression plot for flexion $(\mathrm{R}=0.8417)$ 


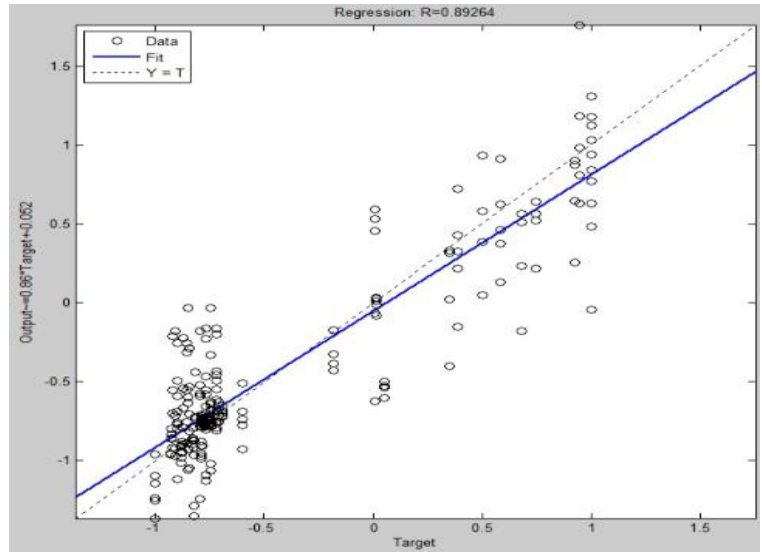

Figure 11(b): Regression plot for extension $(\mathrm{R}=0.8926)$

\section{Discussion and Conclusion} method.

The results show that the joint angles were quite accurately predicted using the artificial neural network

In this study, the accuracy of the EMG signal is of paramount importance in order to properly estimate the joint angles. We have to make sure noise is eliminated by all viable means. A minor change in electrode position causes a major change in the acquired EMG data. Thus an accurate EMG sensor is used. Muscle fatigue is another important factor, so care must be taken to ensure that there is no over-exertion of muscles as this will lead to inaccurate results. Moreover, the network trained is subject specific and may not yield appropriate results if applied on other subject's data. Hence it needs to be re-trained for different subjects.

In summary, this paper develops an offline method for predicting the position and orientation of the arm from surface EMG signals using artificial neural network. The network is trained with EMG feature vector and joint angle values and tested with new EMG data. Some of the estimated results from the network are shown in this paper. Future work will be totrain a Robotic Arm with the Surface EMG signals and then directing it to mimic a human's action of the arm movements. This project can also be extendedto train a realprosthetic arm with the surface EMG signals in order for an amputee to control their own Prosthetic arm through their own EMG signals, rather than using traditional electrical control.

\section{References}

[1] Ajiboye, Weir, "A Heuristic Fuzzy Logic Approach to EMG Pattern Recognition forMultifunctional Prosthesis Control", IEEE TRANSACTIONS ON NEURAL SYSTEMS ANDREHABILITATION ENGINEERING, VOL. 13, NO. 3, SEPTEMBER 2005

[2] Momen, Krishnan, Chau, "Real-Time Classification of Forearm Electromyographic SignalsCorresponding to User-Selected Intentional Movements for Multifunction Prosthesis Control",IEEE TRANSACTIONS ON NEURAL SYSTEMS AND REHABILITATION ENGINEERING,VOL. 15, NO. 4, DECEMBER 2007

[3] Kuiken, Li,Lock, Lipschutz, Miller, Stubblefield, Englehart, "Targeted Muscle Reinnervation for Real-time Myoelectric Control of Multifunction Artificial Arms", 2009 American Medical Association

[4] Srinivasan, DasGupta, Sheng, Chen, "Estimation of Hand Force from Surface Electromyography Signals using Artificial Neural Network", Tenth World Congress on Intelligent Control and Automation, July 6-8, 2012, Beijing, China

[5] "NI-9234 Data Acquisition Board" [Online].Available:http://sine.ni.com/nips/cds/view/p/lang/en/nid/208802

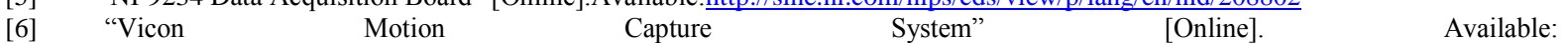
http://www.udel.edu/PT/Research/MAL/essentials_of_motion_capture_v1_2.pdf

[7] "Myoscan-Z EMG Sensor" [Online]. Available:http://thoughttechnology.com/myoscanz.htm

[8]

“LevenbergMarquardtBackpropagationalgorithm”,[Online],Available:http://en.wikipedia.org/wiki/Levenberg\%E2\%80\%93Marquar dt algorithm

"ScaledConjugateGradientbackpropagationalgorithm”,[Online],Available:http://www.ra.cs.unituebingen.de/SNNS/UserManual/n ode242.html

[10 ]"HighBiasandHighVariance",[Online],Available:http://www.statalgo.com/2011/11/09/stanford-ml-5-1-learning-theory-and-thebiasvariancetrade-off

[11] "Kendall ARBO disposable EMG/EKG Electrodes", [Online].Available: http:/ /bio-medical.com/products/kendall-tycoarbodisposable-electrodes-50pkg.html 